\title{
ESHELBY'S INCLUSION AND SCREW DISLOCATION PROBLEMS FOR A THREE-PHASE PIEZOELECTRIC LAMINATE
}

\author{
Xu WAng ${ }^{1}$, Peter Schiavone ${ }^{2 *}$ \\ ${ }^{1}$ School of Mechanical and Power Engineering, East China University of \\ Science and Technology, 130 Meilong Road, Shanghai 200237, China \\ ${ }^{2}$ Department of Mechanical Engineering, University of Alberta, 10-203 \\ Donadeo Innovation Centre for Engineering, Edmonton, Alberta, T6G 1H9, \\ Canada
}

[Received 06 November 2018. Accepted: 18 February 2019] doi: 10.7546/JTAM.49.19.01.07

\begin{abstract}
We propose a simple yet effective method based essentially on analytic continuation to derive analytical solutions for a screw dislocation or a two-dimensional Eshelby inclusion of arbitrary shape in a three-phase piezoelectric laminate composed of two semi-infinite piezoelectric media bonded together via an intermediate piezoelectric layer of finite thickness. The screw dislocation or the Eshelby inclusion can be located either in the upper semiinfinite piezoelectric medium or in the intermediate piezoelectric layer. Elegant expressions for the image force acting on the piezoelectric screw dislocation are presented and compared with existing results. We also observe some very interesting properties of the solutions obtained.
\end{abstract}

KEY WORDS: Three-phase piezoelectric laminate, screw dislocation, Eshelby inclusion, image force, analytic continuation.

\section{INTRODUCTION}

The elastic fields corresponding to stresses and strains induced by dislocations in multilayered structures have been analyzed by many investigators [1-10]. In the majority of these studies, researchers have utilized the so-called 'image method'. The two-dimensional Eshelby's problem of an inclusion of arbitrary shape in multilayered structures, however, has rarely been discussed, except for a number of studies on Eshelby's inclusion problem in bimaterials which use the Green's function method $[11,12]$ or techniques based on analytic continuation and conformal mapping [1317].

In this study, we endeavor to derive the electroelastic field induced by a screw dislocation or a two-dimensional Eshelby inclusion of arbitrary shape in a three-phase

\footnotetext{
${ }^{*}$ Corresponding author e-mail: p.schiavone@ualberta.ca
} 
hexagonal piezoelectric laminate composed of a piezoelectric layer of finite thickness bounded by two semi-infinite piezoelectric media. Rather than employ the traditional image method used by several authors (including those mentioned above), we choose to base our analysis on the technique of analytic continuation $[13,18]$ which we find to be particularly effective in accommodating these types of challenging interaction problems. We derive explicit solutions which, in the case of the dislocation problem, can be conveniently interpreted in terms of image dislocations, image electricpotential-dislocations, image forces and image charges for all three phases of the laminate. General yet elegant expressions of the image force acting on the piezoelectric screw dislocation are obtained using the extended version of the Peach-Koehler formula $[19,20]$ and are compared with existing results in the literature $[1,21,22]$.

\section{BASIC FORMULATION}

In a fixed rectangular coordinate system $x_{i}(i=1,2,3)$, the stresses $\sigma_{i j}$, the strains $\varepsilon_{i j}$, the displacements $u_{i}$, the electric displacements $D_{i}$, the electric fields $E_{i}$ and the electric potential $\varphi$ satisfy the following equations of equilibrium and constitutive relations for an anisotropic piezoelectric solid [23, 24]:

$$
\begin{array}{ll}
\sigma_{i j, j}=0, & D_{i, i}=0, \\
\sigma_{i j}=C_{i j k l} \varepsilon_{k l}-e_{k i j} E_{k}, & D_{k}=e_{k i j} \varepsilon_{i j}+\epsilon_{k l} E_{l}, \\
\varepsilon_{i j}=\frac{1}{2}\left(u_{i, j}+u_{j, i}\right), & E_{i}=-\phi_{i},
\end{array}
$$

where $C_{i j k l}, e_{k i j}$ and $\in_{i j}$ are respectively the elastic stiffnesses, piezoelectric constants and dielectric constants.

For the anti-plane shear deformations of a hexagonal piezoelectric material exhibiting $6 \mathrm{~mm}$ symmetry with its poling direction along the $x_{3}$-axis, the general solution is given by

$$
\begin{gathered}
{\left[\begin{array}{c}
u_{3} \\
\phi
\end{array}\right]=\operatorname{Im}\{\mathbf{f}(z)\},} \\
{\left[\begin{array}{c}
2 \varepsilon_{32}+2 \mathrm{i} \varepsilon_{31} \\
-E_{2}-\mathrm{i} E_{1}
\end{array}\right]=\mathbf{f}^{\prime}(z), \quad\left[\begin{array}{c}
\sigma_{32}+\mathrm{i} \sigma_{31} \\
D_{2}+\mathrm{i} D_{1}
\end{array}\right]=\mathbf{C f}^{\prime}(z),}
\end{gathered}
$$

where $\mathbf{f}(z)$ is a two-dimensional analytic vector function of the complex variable $z=x_{1}+\mathrm{i} x_{2}$ and the real symmetric matrix $\mathbf{C}$ is defined by

$$
\mathbf{C}=\mathbf{C}^{T}=\left[\begin{array}{cc}
C_{44} & e_{15} \\
e_{15} & -\epsilon_{11}
\end{array}\right]
$$


Consider the anti-plane shear deformation of a three-phase piezoelectric laminate composed of two semi-infinite piezoelectric media bonded together through an intermediate piezoelectric layer of finite thickness $h$. Each piezoelectric phase is hexagonal with its poling direction along the $x_{3}$-axis. Let $S_{1}, S_{2}$ and $S_{3}$ denote the upper semi-infinite medium, the intermediate layer and the lower semi-infinite medium, respectively, all of which are perfectly bonded across two planar interfaces at $x_{2}=0$ and $x_{2}=-h$. In what follows, the subscripts 1,2 and 3 are used to identify the respective quantities in $S_{1}, S_{2}$ and $S_{3}$. Here, we assume that a screw dislocation or a two-dimensional Eshelby inclusion of arbitrary shape can be located either in the upper semi-infinite piezoelectric medium or in the intermediate piezoelectric layer of finite thickness.

\section{The General Solution}

The conditions describing continuity of displacement, electric potential, traction and normal electric displacement across the two perfectly bonded interfaces at $x_{2}=0$ and $x_{2}=-h$ can be expressed in terms of the three analytic vector functions $\mathbf{f}_{1}(z), \mathbf{f}_{2}(z)$ and $\mathbf{f}_{3}(z)$ as follows:

$$
\begin{aligned}
& \mathbf{f}_{1}(z)+\overline{\mathbf{f}_{1}(z)}=\boldsymbol{\Gamma}_{1} \mathbf{f}_{2}(z)+\boldsymbol{\Gamma}_{1} \overline{\mathbf{f}_{2}(z)}, \\
& \mathbf{f}_{1}(z)-\overline{\mathbf{f}_{1}(z)}=\mathbf{f}_{2}(z)-\overline{\mathbf{f}_{2}(z)}, \quad \operatorname{Im}\{z\}=0 ; \\
& \mathbf{f}_{2}(z)+\overline{\mathbf{f}_{2}(z)}=\boldsymbol{\Gamma}_{2} \mathbf{f}_{3}(z)+\boldsymbol{\Gamma}_{2} \overline{\mathbf{f}_{3}(z)}, \\
& \mathbf{f}_{2}(z)-\overline{\mathbf{f}_{2}(z)}=\mathbf{f}_{3}(z)-\overline{\mathbf{f}_{3}(z)}, \quad \operatorname{Im}\{z\}=-h,
\end{aligned}
$$

where:

$$
\boldsymbol{\Gamma}_{1}=\mathbf{C}_{1}^{-1} \mathbf{C}_{2}, \quad \boldsymbol{\Gamma}_{2}=\mathbf{C}_{2}^{-1} \mathbf{C}_{3} .
$$

We assume that $\mathbf{f}_{3}(z)$ takes the following general form:

$$
\mathbf{f}_{3}(z)=\mathbf{g}(z),
$$

where $\mathbf{g}(z)$ is to be determined. It will be seen from the ensuing analysis that a judicious choice of $\mathbf{g}(z)$ will be crucial to the successful derivation of the corresponding solutions.

By enforcing the continuity conditions across the two interfaces in Eqs. (7a,7b) and applying the technique of analytic continuation $[13,18]$, we find the following expressions for $\mathbf{f}_{1}(z)$ and $\mathbf{f}_{2}(z)$ :

$$
\mathbf{f}_{2}(z)=\frac{\boldsymbol{\Gamma}_{2}+\mathbf{I}}{2} \mathbf{g}(z)+\frac{\boldsymbol{\Gamma}_{2}-\mathbf{I}}{2} \overline{\mathbf{g}}(z+2 \mathrm{i} h),
$$




$$
\begin{aligned}
\mathbf{f}_{1}(z)= & \frac{\left(\boldsymbol{\Gamma}_{1}+\mathbf{I}\right)\left(\boldsymbol{\Gamma}_{2}+\mathbf{I}\right)}{4} \mathbf{g}(z)+\frac{\left(\boldsymbol{\Gamma}_{1}-\mathbf{I}\right)\left(\boldsymbol{\Gamma}_{2}-\mathbf{I}\right)}{4} \mathbf{g}(z-2 \mathrm{i} h) \\
& +\frac{\left(\boldsymbol{\Gamma}_{1}-\mathbf{I}\right)\left(\boldsymbol{\Gamma}_{2}+\mathbf{I}\right)}{4} \overline{\mathbf{g}}(z)+\frac{\left(\boldsymbol{\Gamma}_{1}+\mathbf{I}\right)\left(\boldsymbol{\Gamma}_{2}-\mathbf{I}\right)}{4} \overline{\mathbf{g}}(z+2 \mathrm{i} h) .
\end{aligned}
$$

We remark that the obtained general solution is thus strikingly simple.

\section{The SCREw Dislocation Problem}

In this section, we first consider a screw dislocation in the three-phase piezoelectric laminate. The screw dislocation suffers a jump $b_{3}$ in anti-plane displacement and a jump $\Delta \phi$ in electric potential across the slip plane. Meanwhile, it is subjected to a line force $p$ and a line charge $q$ at its core. The screw dislocation can be located either at $z=\mathrm{i} d(d>0)$ in the upper semi-infinite medium (phase 1) or located at $z=-\mathrm{i} d$ $(0<d<h)$ in the intermediate layer (phase 2).

\subsection{DISLOCATION IN THE UPPER SEMI-INFINITE PIEZOELECTRIC MEDIUM (PHASE 1)}

When the piezoelectric screw dislocation is located in the upper semi-infinite piezoelectric medium (phase 1), a careful examination of Eq. (11) reveals that $\mathbf{g}(z)$ should take the following form:

$$
\mathbf{g}(z)=\frac{2}{\pi} \sum_{n=0}^{+\infty} \ln [z-\mathrm{i}(d+2 n h)] \boldsymbol{\Lambda}^{n}\left(\boldsymbol{\Gamma}_{2}+\mathbf{I}\right)^{-1}\left(\boldsymbol{\Gamma}_{1}+\mathbf{I}\right)^{-1}\left(\hat{\mathbf{b}}-\mathrm{iC}_{1}^{-1} \hat{\mathbf{f}}\right)
$$

where $\mathbf{I}$ is the $2 \times 2$ identity matrix, and

$$
\begin{gathered}
\boldsymbol{\Lambda}=-\left(\boldsymbol{\Gamma}_{2}+\mathbf{I}\right)^{-1}\left(\boldsymbol{\Gamma}_{1}+\mathbf{I}\right)^{-1}\left(\boldsymbol{\Gamma}_{1}-\mathbf{I}\right)\left(\boldsymbol{\Gamma}_{2}-\mathbf{I}\right), \\
\hat{\mathbf{b}}=\left[\begin{array}{ll}
b_{3} & \Delta \phi
\end{array}\right]^{T}, \quad \hat{\mathbf{f}}=\left[\begin{array}{ll}
p & -q
\end{array}\right]^{T} .
\end{gathered}
$$

Remark 1. By choosing Eq. (12), $\mathbf{f}_{1}(z)$ will exhibit the singular behavior

$$
\mathbf{f}_{1}(z) \cong \frac{1}{2 \pi} \ln (z-\mathrm{i} d)\left(\hat{\mathbf{b}}-\mathrm{iC}_{1}^{-1} \hat{\mathbf{f}}\right)+O(1) \quad \text { as } \quad z \rightarrow \mathrm{i} d
$$

and is analytic elsewhere.

Substituting Eq. (12) into Eqs. (9)-(11), we finally obtain the following explicit expressions for $\mathbf{f}_{1}(z), \mathbf{f}_{2}(z)$ and $\mathbf{f}_{3}(z)$ :

$$
\mathbf{f}_{1}(z)=\frac{\ln (z-\mathrm{i} d)}{2 \pi}\left(\hat{\mathbf{b}}-\mathrm{i} \mathbf{C}_{1}^{-1} \hat{\mathbf{f}}\right)+\frac{1}{2 \pi} \sum_{n=0}^{+\infty} \ln [z+\mathrm{i}(d+2 n h)]
$$


Eshelby's Inclusion and Screw Dislocation Problems for a Three-Phase...

$$
\begin{gathered}
\times\left(\boldsymbol{\Gamma}_{1}-\mathbf{I}\right)\left(\boldsymbol{\Gamma}_{2}+\mathbf{I}\right) \boldsymbol{\Lambda}^{n}\left(\boldsymbol{\Gamma}_{2}+\mathbf{I}\right)^{-1}\left(\boldsymbol{\Gamma}_{1}+\mathbf{I}\right)^{-1}\left(\hat{\mathbf{b}}+\mathrm{i} \mathbf{C}_{1}^{-1} \hat{\mathbf{f}}\right) \\
+\frac{1}{2 \pi} \sum_{n=0}^{+\infty} \ln [z+\mathrm{i}(d+2 h(n+1))] \\
\times\left(\boldsymbol{\Gamma}_{1}+\mathbf{I}\right)\left(\boldsymbol{\Gamma}_{2}-\mathbf{I}\right) \boldsymbol{\Lambda}^{n}\left(\boldsymbol{\Gamma}_{2}+\mathbf{I}\right)^{-1}\left(\boldsymbol{\Gamma}_{1}+\mathbf{I}\right)^{-1}\left(\hat{\mathbf{b}}+\mathrm{i} \mathbf{C}_{1}^{-1} \hat{\mathbf{f}}\right), \\
\mathbf{f}_{2}(z)=\frac{1}{\pi} \sum_{n=0}^{+\infty} \ln [z-\mathrm{i}(d+2 n h)]\left(\boldsymbol{\Gamma}_{2}+\mathbf{I}\right) \boldsymbol{\Lambda}^{n}\left(\boldsymbol{\Gamma}_{2}+\mathbf{I}\right)^{-1}\left(\boldsymbol{\Gamma}_{1}+\mathbf{I}\right)^{-1}\left(\hat{\mathbf{b}}-\mathrm{i} \mathbf{C}_{1}^{-1} \hat{\mathbf{f}}\right) \\
+\frac{1}{\pi} \sum_{n=0}^{+\infty} \ln [z+\mathrm{i}(d+2 h(n+1))] \\
\times\left(\boldsymbol{\Gamma}_{2}-\mathbf{I}\right) \boldsymbol{\Lambda}^{n}\left(\boldsymbol{\Gamma}_{2}+\mathbf{I}\right)^{-1}\left(\boldsymbol{\Gamma}_{1}+\mathbf{I}\right)^{-1}\left(\hat{\mathbf{b}}+\mathrm{i} \mathbf{C}_{1}^{-1} \hat{\mathbf{f}}\right), \\
\mathbf{f}_{3}(z)=\frac{2}{\pi} \sum_{n=0}^{+\infty} \ln [z-\mathrm{i}(d+2 n h)] \boldsymbol{\Lambda}^{n}\left(\boldsymbol{\Gamma}_{2}+\mathbf{I}\right)^{-1}\left(\boldsymbol{\Gamma}_{1}+\mathbf{I}\right)^{-1}\left(\hat{\mathbf{b}}-\mathrm{iC}_{1}^{-1} \hat{\mathbf{f}}\right) .
\end{gathered}
$$

The above solution can be conveniently interpreted in terms of image dislocations, image electric-potential-dislocations, image forces and image charges for all three phases. By using the extended version of the Peach-Koehler formula [19, 20], an elegant and concise expression for the image force acting on the piezoelectric screw dislocation can be finally obtained as:

$$
F_{1}=0, \quad F_{2}=\frac{1}{4 \pi}\left(\hat{\mathbf{b}}^{T} \mathbf{C}_{1} \mathbf{M} \hat{\mathbf{b}}+\hat{\mathbf{f}}^{T} \mathbf{M} \mathbf{C}_{1}^{-1} \hat{\mathbf{f}}\right),
$$

where $F_{1}$ and $F_{2}$ are respectively the force components along the $x_{1}$ and $x_{2}$ directions and the $2 \times 2$ real matrix $\mathbf{M}$ is defined by

$$
\begin{aligned}
\mathbf{M}= & \frac{1}{d}\left(\boldsymbol{\Gamma}_{1}-\mathbf{I}\right)\left(\boldsymbol{\Gamma}_{1}+\mathbf{I}\right)^{-1}+\left[\boldsymbol{\Gamma}_{1}+\mathbf{I}-\left(\boldsymbol{\Gamma}_{1}-\mathbf{I}\right)\left(\boldsymbol{\Gamma}_{1}+\mathbf{I}\right)^{-1}\left(\boldsymbol{\Gamma}_{1}-\mathbf{I}\right)\right] \\
& \times\left(\boldsymbol{\Gamma}_{2}-\mathbf{I}\right) \sum_{n=1}^{+\infty} \frac{1}{d+n h} \boldsymbol{\Lambda}^{n-1}\left(\boldsymbol{\Gamma}_{2}+\mathbf{I}\right)^{-1}\left(\boldsymbol{\Gamma}_{1}+\mathbf{I}\right)^{-1}
\end{aligned}
$$

It can be verified that both $\mathbf{C}_{1} \mathbf{M}$ and $\mathbf{M} \mathbf{C}_{1}^{-1}$ are symmetric.

When $h \rightarrow \infty$, Eq. (16) becomes:

$$
F_{2}=\frac{1}{4 \pi d}\left[\hat{\mathbf{b}}^{T} \mathbf{C}_{1}\left(\boldsymbol{\Gamma}_{1}-\mathbf{I}\right)\left(\boldsymbol{\Gamma}_{1}+\mathbf{I}\right)^{-1} \hat{\mathbf{b}}+\hat{\mathbf{f}}^{T}\left(\boldsymbol{\Gamma}_{1}-\mathbf{I}\right)\left(\boldsymbol{\Gamma}_{1}+\mathbf{I}\right)^{-1} \mathbf{C}_{1}^{-1} \hat{\mathbf{f}}\right]
$$

When $h \rightarrow 0$, Eq. (16) becomes: 


$$
\begin{aligned}
F_{2}=\frac{1}{4 \pi d}\left[\hat { \mathbf { b } } ^ { T } \mathbf { C } _ { 1 } ( \boldsymbol { \Gamma } _ { 1 } \boldsymbol { \Gamma } _ { 2 } - \mathbf { I } ) \left(\boldsymbol{\Gamma}_{1} \boldsymbol{\Gamma}_{2}\right.\right. & +\mathbf{I})^{-1} \hat{\mathbf{b}} \\
& \left.+\hat{\mathbf{f}}^{T}\left(\boldsymbol{\Gamma}_{1} \boldsymbol{\Gamma}_{2}-\mathbf{I}\right)\left(\boldsymbol{\Gamma}_{1} \boldsymbol{\Gamma}_{2}+\mathbf{I}\right)^{-1} \mathbf{C}_{1}^{-1} \hat{\mathbf{f}}\right]
\end{aligned}
$$

Both Eqs. (18) and (19) agree with existing results for a piezoelectric screw dislocation interacting with a perfect bimaterial interface $[21,22]$.

When all three phases are dielectric elastic or non-piezoelectric materials with their piezoelectric constants set to zero and $\Delta \phi=p=q=0$, Eq. (16) will simply reduce to the classical solution obtained by Chou [1].

\subsection{DisLOCATION IN THE INTERMEDIATE PIEZOELECTRIC LAYER (PHASE 2)}

When the piezoelectric screw dislocation is located in the intermediate piezoelectric layer (phase 2), a careful examination of Eqs. (10) and (11) reveals that $\mathbf{g}(z)$ should take the following form:

$$
\begin{gathered}
\mathbf{g}(z)=\frac{1}{\pi} \sum_{n=0}^{+\infty} \ln [z-\mathrm{i}(-d+2 n h)] \mathbf{\Lambda}^{n}\left(\boldsymbol{\Gamma}_{2}+\mathbf{I}\right)^{-1}\left(\hat{\mathbf{b}}-\mathrm{i} \mathbf{C}_{2}^{-1} \hat{\mathbf{f}}\right) \\
-\frac{1}{\pi} \sum_{n=0}^{+\infty} \ln [z-\mathrm{i}(d+2 n h)] \boldsymbol{\Lambda}^{n}\left(\boldsymbol{\Gamma}_{2}+\mathbf{I}\right)^{-1}\left(\boldsymbol{\Gamma}_{1}+\mathbf{I}\right)^{-1}\left(\boldsymbol{\Gamma}_{1}-\mathbf{I}\right)\left(\hat{\mathbf{b}}+\mathrm{i} \mathbf{C}_{2}^{-1} \hat{\mathbf{f}}\right) .
\end{gathered}
$$

Remark 2. By choosing Eq. (20), $\mathbf{f}_{2}(z)$ will exhibit the singular behavior

$$
\mathbf{f}_{2}(z) \cong \frac{1}{2 \pi} \ln (z+\mathrm{i} d)\left(\hat{\mathbf{b}}-\mathrm{iC}_{2}^{-1} \hat{\mathbf{f}}\right)+O(1) \quad \text { as } \quad z \rightarrow-\mathrm{i} d
$$

whilst $\mathbf{f}_{1}(z)$ is analytic everywhere in its domain of definition.

By substituting Eq. (20) into Eqs. (9)-(11), we finally obtain the following explicit expressions for $\mathbf{f}_{1}(z), \mathbf{f}_{2}(z)$ and $\mathbf{f}_{3}(z)$

$$
\text { (21a) } \begin{aligned}
\mathbf{f}_{1}(z)= & \frac{\ln (z+\mathrm{i} d)}{4 \pi}\left(\boldsymbol{\Gamma}_{1}+\mathbf{I}\right)\left(\hat{\mathbf{b}}-\mathrm{i} \mathbf{C}_{2}^{-1} \hat{\mathbf{f}}\right) \\
& +\frac{1}{4 \pi} \sum_{n=1}^{+\infty} \ln [z+\mathrm{i}(-d+2 n h)] \\
& \times\left(\boldsymbol{\Gamma}_{1}-\mathbf{I}\right)\left(\boldsymbol{\Gamma}_{2}+\mathbf{I}\right) \boldsymbol{\Lambda}^{n}\left(\boldsymbol{\Gamma}_{2}+\mathbf{I}\right)^{-1}\left(\hat{\mathbf{b}}+\mathrm{iC}_{2}^{-1} \hat{\mathbf{f}}\right) \\
& -\frac{1}{4 \pi} \sum_{n=0}^{+\infty} \ln [z+\mathrm{i}(d+2 n h)]
\end{aligned}
$$


Eshelby's Inclusion and Screw Dislocation Problems for a Three-Phase...

$$
\begin{aligned}
& \times\left(\boldsymbol{\Gamma}_{1}-\mathbf{I}\right)\left(\boldsymbol{\Gamma}_{2}+\mathbf{I}\right) \mathbf{\Lambda}^{n}\left(\boldsymbol{\Gamma}_{2}+\mathbf{I}\right)^{-1}\left(\boldsymbol{\Gamma}_{1}+\mathbf{I}\right)^{-1}\left(\boldsymbol{\Gamma}_{1}-\mathbf{I}\right)\left(\hat{\mathbf{b}}-\mathrm{i} \mathbf{C}_{2}^{-1} \hat{\mathbf{f}}\right) \\
& +\frac{1}{4 \pi} \sum_{n=0}^{+\infty} \ln [z+\mathrm{i}(-d+2 h(n+1))] \\
& \times\left(\boldsymbol{\Gamma}_{1}+\mathbf{I}\right)\left(\boldsymbol{\Gamma}_{2}-\mathbf{I}\right) \boldsymbol{\Lambda}^{n}\left(\boldsymbol{\Gamma}_{2}+\mathbf{I}\right)^{-1}\left(\hat{\mathbf{b}}+\mathrm{i} \mathbf{C}_{2}^{-1} \hat{\mathbf{f}}\right) \\
& -\frac{1}{4 \pi} \sum_{n=0}^{+\infty} \ln [z+\mathrm{i}(d+2 h(n+1))] \\
& \times\left(\boldsymbol{\Gamma}_{1}+\mathbf{I}\right)\left(\boldsymbol{\Gamma}_{2}-\mathbf{I}\right) \Lambda^{n}\left(\boldsymbol{\Gamma}_{2}+\mathbf{I}\right)^{-1}\left(\boldsymbol{\Gamma}_{1}+\mathbf{I}\right)^{-1}\left(\boldsymbol{\Gamma}_{1}-\mathbf{I}\right)\left(\hat{\mathbf{b}}-\mathrm{iC}_{2}^{-1} \hat{\mathbf{f}}\right),
\end{aligned}
$$

(21b)

$$
\begin{aligned}
\mathbf{f}_{2}(z)= & \frac{1}{2 \pi} \sum_{n=0}^{+\infty} \ln [z-\mathrm{i}(-d+2 n h)]\left(\boldsymbol{\Gamma}_{2}+\mathbf{I}\right) \boldsymbol{\Lambda}^{n}\left(\boldsymbol{\Gamma}_{2}+\mathbf{I}\right)^{-1}\left(\hat{\mathbf{b}}-\mathrm{i} \mathbf{C}_{2}^{-1} \hat{\mathbf{f}}\right) \\
& -\frac{1}{2 \pi} \sum_{n=0}^{+\infty} \ln [z-\mathrm{i}(d+2 n h)] \\
& \times\left(\boldsymbol{\Gamma}_{2}+\mathbf{I}\right) \boldsymbol{\Lambda}^{n}\left(\boldsymbol{\Gamma}_{2}+\mathbf{I}\right)^{-1}\left(\boldsymbol{\Gamma}_{1}+\mathbf{I}\right)^{-1}\left(\boldsymbol{\Gamma}_{1}-\mathbf{I}\right)\left(\hat{\mathbf{b}}+\mathrm{i} \mathbf{C}_{2}^{-1} \hat{\mathbf{f}}\right) \\
+ & \frac{1}{2 \pi} \sum_{n=0}^{+\infty} \ln [z+\mathrm{i}(-d+2 h(n+1))] \\
\times & \left(\boldsymbol{\Gamma}_{2}-\mathbf{I}\right) \boldsymbol{\Lambda}^{n}\left(\boldsymbol{\Gamma}_{2}+\mathbf{I}\right)^{-1}\left(\hat{\mathbf{b}}+\mathrm{i} \mathbf{C}_{2}^{-1} \hat{\mathbf{f}}\right) \\
- & \frac{1}{2 \pi} \sum_{n=0}^{+\infty} \ln [z+\mathrm{i}(d+2 h(n+1))] \\
\times & \left(\boldsymbol{\Gamma}_{2}-\mathbf{I}\right) \boldsymbol{\Lambda}^{n}\left(\boldsymbol{\Gamma}_{2}+\mathbf{I}\right)^{-1}\left(\boldsymbol{\Gamma}_{1}+\mathbf{I}\right)^{-1}\left(\boldsymbol{\Gamma}_{1}-\mathbf{I}\right)\left(\hat{\mathbf{b}}-\mathrm{i} \mathbf{C}_{2}^{-1} \hat{\mathbf{f}}\right), \\
\mathbf{f}_{3}(z)= & \frac{1}{\pi} \sum_{n=0}^{+\infty} \ln [z-\mathrm{i}(-d+2 n h)] \boldsymbol{\Lambda}^{n}\left(\boldsymbol{\Gamma}_{2}+\mathbf{I}\right)^{-1}\left(\hat{\mathbf{b}}-\mathrm{i} \mathbf{C}_{2}^{-1} \hat{\mathbf{f}}\right) \\
& \quad-\frac{1}{\pi} \sum_{n=0}^{+\infty} \ln [z-\mathrm{i}(d+2 n h)] \\
& \times \boldsymbol{\Lambda}^{n}\left(\boldsymbol{\Gamma}_{2}+\mathbf{I}\right)^{-1}\left(\boldsymbol{\Gamma}_{1}+\mathbf{I}\right)^{-1}\left(\boldsymbol{\Gamma}_{1}-\mathbf{I}\right)\left(\hat{\mathbf{b}}+\mathrm{i} \mathbf{C}_{2}^{-1} \hat{\mathbf{f}}\right) .
\end{aligned}
$$

It is again particularly convenient to interpret the above solution in terms of image dislocations, image electric-potential-dislocations, image forces and image charges for all the three phases. By using the extended version of the Peach-Koehler formula, the image force acting on the piezoelectric screw dislocation is then derived as

$$
F_{1}=0, \quad F_{2}=\frac{1}{4 \pi}\left(\hat{\mathbf{b}}^{T} \mathbf{C}_{2} \mathbf{N} \hat{\mathbf{b}}+\hat{\mathbf{f}}^{T} \mathbf{N} \mathbf{C}_{2}^{-1} \hat{\mathbf{f}}\right),
$$


where the $2 \times 2$ real matrix $\mathbf{N}$ is defined by

$$
\begin{aligned}
\mathbf{N}= & \frac{1}{d}\left(\boldsymbol{\Gamma}_{1}+\mathbf{I}\right)^{-1}\left(\boldsymbol{\Gamma}_{1}-\mathbf{I}\right)+\sum_{n=1}^{+\infty} \frac{1}{n h-d}\left(\boldsymbol{\Gamma}_{2}-\mathbf{I}\right) \boldsymbol{\Lambda}^{n-1}\left(\boldsymbol{\Gamma}_{2}+\mathbf{I}\right)^{-1} \\
& +\sum_{n=1}^{+\infty} \frac{1}{n h+d}\left(\boldsymbol{\Gamma}_{2}+\mathbf{I}\right) \Lambda^{n}\left(\boldsymbol{\Gamma}_{2}+\mathbf{I}\right)^{-1}\left(\boldsymbol{\Gamma}_{1}+\mathbf{I}\right)^{-1}\left(\boldsymbol{\Gamma}_{1}-\mathbf{I}\right)
\end{aligned}
$$

It can be verified that both $\mathbf{C}_{2} \mathbf{N}$ and $\mathbf{N C}_{2}^{-1}$ are symmetric. During the derivation of Eq. (22), we have used the property that both the two matrices

$$
\begin{aligned}
& \mathbf{C}_{2}\left[\left(\boldsymbol{\Gamma}_{2}-\mathbf{I}\right) \boldsymbol{\Lambda}^{n}\left(\boldsymbol{\Gamma}_{2}-\mathbf{I}\right)^{-1}-\left(\boldsymbol{\Gamma}_{2}+\mathbf{I}\right) \boldsymbol{\Lambda}^{n}\left(\boldsymbol{\Gamma}_{2}+\mathbf{I}\right)^{-1}\right] \text { and } \\
& {\left[\left(\boldsymbol{\Gamma}_{2}-\mathbf{I}\right) \boldsymbol{\Lambda}^{n}\left(\boldsymbol{\Gamma}_{2}-\mathbf{I}\right)^{-1}-\left(\boldsymbol{\Gamma}_{2}+\mathbf{I}\right) \boldsymbol{\Lambda}^{n}\left(\boldsymbol{\Gamma}_{2}+\mathbf{I}\right)^{-1}\right] \mathbf{C}_{2}^{-1}}
\end{aligned}
$$

are anti-symmetric.

When all three phases are dielectric elastic with their piezoelectric constants set to zero and $\Delta \phi=p=q=0$, Eq. (22) will again simply reduce to the corresponding classical solution by Chou [1].

\section{The EShelby InClusion Problem}

Here, the three-phase piezoelectric laminate contains a two-dimensional Eshelby inclusion of arbitrary shape (simply connected inclusion enclosed by a simple curve [18]) undergoing uniform anti-plane eigenstrains $\left(\varepsilon_{13}^{*}, \varepsilon_{23}^{*}\right)$ and in-plane eigenelectric fields $\left(E_{1}^{*}, E_{2}^{*}\right)$. The elastic, piezoelectric and dielectric constants for the Eshelby inclusion and its surrounding material are taken to be identical. The Eshelby inclusion can be located either within the upper semi-infinite medium or within the intermediate layer.

\subsection{AN ESHELBY INCLUSION IN THE UPPER SEMI-INFINITE PIEZOELECTRIC MEDIUM (PHASE 1)}

When the Eshelby inclusion lies in the upper semi-infinite piezoelectric medium, the continuity conditions across the perfectly bonded interface $L$ between the Eshelby inclusion and its surrounding material are described by

$$
\begin{aligned}
& \mathbf{f}_{1}(z)+\overline{\mathbf{f}_{1}(z)}=\mathbf{f}_{0}(z)+\overline{\mathbf{f}_{0}(z)}, \\
& \mathbf{f}_{1}(z)-\overline{\mathbf{f}_{1}(z)}=\mathbf{f}_{0}(z)-\overline{\mathbf{f}_{0}(z)}+2 \mathbf{v} z-2 \overline{\mathbf{v}} \bar{z}, \quad z \in L,
\end{aligned}
$$

where $\mathbf{f}_{0}(z)$ is the analytic vector function defined inside the Eshelby inclusion denoted here by $S_{0}, \mathbf{f}_{1}(z)$ is the analytic vector function defined in the upper half-plane 
outside the inclusion here denoted by $S_{1}^{\prime}$, and the two-dimensional complex vector $\mathbf{v}$ is defined in terms of the eigenstrains and eigenelectric fields as follows:

$$
\mathbf{v}=\left[\begin{array}{c}
\varepsilon_{23}^{*}+\mathrm{i} \varepsilon_{13}^{*} \\
-\frac{1}{2}\left(E_{2}^{*}+\mathrm{i} E_{1}^{*}\right)
\end{array}\right] .
$$

Adding the two conditions in Eq. (24), we finally obtain

$$
\mathbf{f}_{1}(z)+\overline{\mathbf{v}}[D(z)-P(z)]=\mathbf{f}_{0}(z)+\mathbf{v} z-\overline{\mathbf{v}} P(z), \quad z \in L,
$$

where $D(z)=\bar{z}, z \in L$ and $D(z) \cong P(z)+O\left(z^{-1}\right),|z| \rightarrow \infty$ [18]. It is clear that the term $[D(z)-P(z)]$ is analytic in the exterior of the Eshelby inclusion including at infinity. An explicit expression for $[D(z)-P(z)]$ in the case of an elliptical inclusion was obtained by $\mathrm{Ru}[18]$.

In view of Eq. (26), we can construct the following auxiliary vector function $\Omega(z)$ :

$$
\boldsymbol{\Omega}(z)=\left\{\begin{array}{ll}
\mathbf{f}_{1}(z)+\overline{\mathbf{v}}[D(z)-P(z)], & z \in S_{1}^{\prime} \\
\mathbf{f}_{0}(z)+\mathbf{v} z-\overline{\mathbf{v}} P(z), & z \in S_{0}
\end{array} .\right.
$$

It is seen that $\Omega(z)$ is continuous across $L$ and then analytic in $S_{1}=S_{1}^{\prime} \cup S_{0}$ including the point at infinity. It is sufficient to treat the term $-\overline{\mathbf{v}}[D(z)-P(z)]$ as the singular (or principal) part of $\mathbf{f}_{1}(z)$. As a result, an examination of Eq. (11) reveals that $\mathbf{g}(z)$ should take the following form:

$$
\mathbf{g}(z)=-4 \sum_{n=0}^{+\infty}[D(z-2 \mathrm{i} n h)-P(z-2 \mathrm{i} n h)] \boldsymbol{\Lambda}^{n}\left(\boldsymbol{\Gamma}_{2}+\mathbf{I}\right)^{-1}\left(\boldsymbol{\Gamma}_{1}+\mathbf{I}\right)^{-1} \overline{\mathbf{v}}
$$

Substituting the above into Eqs. (9)-(11), we finally obtain the three analytic vector functions $\mathbf{f}_{1}(z), \mathbf{f}_{2}(z)$ and $\mathbf{f}_{3}(z)$, whose specific expressions are not given here for reasons of brevity. Furthermore, $\mathbf{f}_{0}(z)$ can be obtained from Eq. (27) as follows:

$$
\mathbf{f}_{0}(z)=\mathbf{f}_{1}(z)+\overline{\mathbf{v}} D(z)-\mathbf{v} z .
$$

\subsection{AN ESHELBY INCLUSION IN THE INTERMEDIATE PIEZOELECTRIC LAYER (PHASE 2)}

When the Eshelby inclusion is located in the intermediate piezoelectric layer, the continuity conditions across the perfect interface $L$ between the Eshelby inclusion and its surrounding material are described by

$$
\begin{aligned}
& \mathbf{f}_{2}(z)+\overline{\mathbf{f}_{2}(z)}=\mathbf{f}_{0}(z)+\overline{\mathbf{f}_{0}(z)}, \\
& \mathbf{f}_{2}(z)-\overline{\mathbf{f}_{2}(z)}=\mathbf{f}_{0}(z)-\overline{\mathbf{f}_{0}(z)}+2 \mathbf{v} z-2 \overline{\mathbf{v}} \bar{z}, \quad z \in L,
\end{aligned}
$$


where $\mathbf{f}_{0}(z)$ is the analytic vector function defined in the Eshelby inclusion denoted again by $S_{0}$ and $\mathbf{f}_{2}(z)$ is the analytic vector function defined in the intermediate layer outside the inclusion denoted by $S_{2}^{\prime}$. The complex vector $\mathbf{v}$ has been defined in Eq. (25).

Adding the two conditions in Eq. (30), we finally arrive at:

$$
\mathbf{f}_{2}(z)+\overline{\mathbf{v}}[D(z)-P(z)]=\mathbf{f}_{0}(z)+\mathbf{v} z-\overline{\mathbf{v}} P(z), \quad z \in L .
$$

Considering the above equation, we can construct the following auxiliary vector function $\boldsymbol{\Psi}(z)$

$$
\boldsymbol{\Psi}(z)=\left\{\begin{array}{ll}
\mathbf{f}_{2}(z)+\overline{\mathbf{v}}[D(z)-P(z)], & z \in S_{2}^{\prime} \\
\mathbf{f}_{0}(z)+\mathbf{v} z-\overline{\mathbf{v}} P(z), & z \in S_{0}
\end{array} .\right.
$$

It is seen that $\boldsymbol{\Psi}(z)$ is continuous across $L$ and then analytic in $S_{2}=S_{2}^{\prime} \cup S_{0}$ including the point at infinity. It is sufficient to treat the term $-\overline{\mathbf{v}}[D(z)-P(z)]$ as the singular (or principal) part of $\mathbf{f}_{2}(z)$. As a result, an examination of Eqs. (10) and (11) reveals that $\mathrm{g}(z)$ should take the following form:

$$
\begin{aligned}
& \mathbf{g}(z)=-2 \sum_{n=0}^{+\infty}[D(z-2 \mathrm{i} n h)-P(z-2 \mathbf{i} n h)] \mathbf{\Lambda}^{n}\left(\boldsymbol{\Gamma}_{2}+\mathbf{I}\right)^{-1} \overline{\mathbf{v}} \\
& +2 \sum_{n=0}^{+\infty}[\bar{D}(z-2 \mathrm{i} n h)-\bar{P}(z-2 \mathrm{i} n h)] \boldsymbol{\Lambda}^{n}\left(\boldsymbol{\Gamma}_{2}+\mathbf{I}\right)^{-1}\left(\boldsymbol{\Gamma}_{1}+\mathbf{I}\right)^{-1}\left(\boldsymbol{\Gamma}_{1}-\mathbf{I}\right) \mathbf{v}
\end{aligned}
$$

Substituting the above into Eqs. (9)-(11), we finally obtain the three analytic vector functions $\mathbf{f}_{1}(z), \mathbf{f}_{2}(z)$ and $\mathbf{f}_{3}(z)$, the specific expressions of which are again suppressed here for sake of brevity. Furthermore, $\mathbf{f}_{0}(z)$ can be obtained from Eq. (32) as follows:

$$
\mathbf{f}_{0}(z)=\mathbf{f}_{2}(z)+\overline{\mathbf{v}} D(z)-\mathbf{v} z .
$$

\section{CONCLUSIONS}

Using the technique of analytic continuation, analytical solutions have been successfully derived for a screw dislocation or a two-dimensional Eshelby inclusion of arbitrary shape in any of the phases of a three-phase piezoelectric laminate consisting of an intermediate piezoelectric layer sandwiched between two semi-infinite piezoelectric media. Our analysis clearly indicates that the crucial step lies in the judicious choice of the analytic vector function $\mathrm{g}(z)$. Finally, we mention here that the extension of the present method to plane deformations encounters certain difficulties 
essentially resulting from the presence of both the complex potential $\varphi(z)$ and its derivative $\varphi^{\prime}(z)$ in the corresponding expressions for displacements and the resultant force [18]. The derivative of the complex potential will persist despite the definition of a new complex potential [13] in view of the fact that there are two interfaces (not one) in the case of a three-phase laminate.

\section{ACKNOWLEDGEMENTS}

This work is supported by the National Natural Science Foundation of China (Grant No. 11272121) and through a Discovery Grant from the Natural Sciences and Engineering Research Council of Canada (Grant No: RGPIN-2017-03716115112).

\section{REFERENCES}

[1] Y.T. CHOU (1966) Screw Dislocations in and Near Lamellar Inclusions. Physica Status Solidi (b) 17 509-516.

[2] L.S. Lin, Y.T. ChOU (1975) Screw Dislocations in a Three-Phase Anisotropic Medium. International Journal of Engineering Science 13 317-325.

[3] S.V. Kamat, J.P. HiRTh, B. CARnahan (1987) Image Forces on Screw Dislocations in Multilayer Structures. Scripta Metallurgica 21 1587-1592.

[4] M.L. ÖveçoĞLu, M.F. Doerner, W.D. Nix. Elastic interactions of screw dislocations in thin films on substrates. Acta Metallurgica 35 294-2957.

[5] L. Stagni, R. Lizzio (1992) Interaction between an Edge Dislocation and a Lamellar Inhomogeneity with a Slipping Interface. Journal of Applied Mechanics 59 215-217.

[6] C.C. MA, R.L. Lin (2002) Full-Field Analysis of a Planar Anisotropic Layered HalfPlane for Concentrated Forces and Edge Dislocations. Proceedings: Mathematical, Physical and Engineering Sciences 458 2369-2392.

[7] C.C. MA, H.T. LU (2006) Theoretical Analysis of Screw Dislocations and Image Forces in Anisotropic Multilayered Media. Physical Review B 73 144102(1)144102(12).

[8] K. Zhou, M.S. Wu (2010) Elastic Fields Due to an Edge Dislocation in an Isotropic Film-Substrate by the Image Method. Acta Mechanica 211 271-292.

[9] X. WANG (2011) On Edge Dislocation in a Multilayered Laminate. Theoretical and Applied Mechanics Letters 1 021005(1)-021005(4).

[10] N. Ogbonna (2014) Force on a Screw Dislocation in a Laminated Structure. Mathematics and Mechanics of Solids 19 694-702.

[11] H.T. Zhang, Y.T. ChOU (1985) Antiplane Eigenstrain Problem of an Elliptic Inclusion in a Two-Phase Anisotropic Medium. ASME Journal of Applied Mechanics 52 87-90.

[12] H.Y. YU, S.C. SandaY (1991) Elastic Field in Jointed Semi-Infinite Solids with an Inclusion. Proceedings of the Royal Society of London Series A 434 521-530. 
[13] Z.G. SuO (1989) Singularities Interacting with Interfaces and Cracks. International Journal of Solids and Structures 25 1133-1142.

[14] C.Q. Ru (2001) A Two-Dimensional Eshelby Problem for Two Bonded Piezoelectric Half-Planes. Proceedings of the Royal Society of London Series A 457 865-883.

[15] C.Q. Ru, P. Schiavone, A. Mioduchowski (2001) Elastic Fields in Two Jointed Half-Planes with an Inclusion of Arbitrary Shape. Zeitschrift fur Angewandte Mathematik und Physik 52 18-32.

[16] X. Wang, L.J. SudaK, C.Q. RU (2007) Elastic Fields in Two Imperfectly Bonded Half-Planes with a Thermal Inclusion of Arbitrary Shape. Zeitschrift fur Angewandte Mathematik und Physik 58 488-509.

[17] X. Wang, E. Pan (2010) Two-Dimensional Eshelby'S Problem for Two Imperfectly Bonded Piezoelectric Half-Planes. International Journal of Solids and Structures 47 148-160.

[18] C.Q. RU (1999) Analytic Solution for Eshelby's Problem of an Inclusion of Arbitrary Shape in a Plane or Half-Plane. ASME Journal of Applied Mechanics 66 315-322.

[19] Y.E. PAK (1990) Force on a Piezoelectric Screw Dislocation. ASME Journal of Applied Mechanics $\mathbf{5 7}$ 863-869.

[20] K.Y. LeE, W.G. LeE, Y.E. PAK (2000) Interaction between a Semi-Infinite Crack and a Screw Dislocation in a Piezoelectric Material. ASME Journal of Applied Mechanics 67 165-170.

[21] J.X. LiU, S.Y. DU, B. WANG (1999) A Screw Dislocation Interacting with a Piezoelectric Bimaterial Interface. Mechanics Research Communications 26 415-420.

[22] X. WANG, H. FAN (2015) A Piezoelectric Screw Dislocation in a Bimaterial with Surface Piezoelectricity. Acta Mechanica 226 3317-3331.

[23] Z. Suo, C.M. Kuo, D.M. BARnetT, J.R. Willis (1992) Fracture Mechanics for Piezoelectric Ceramics. Journal of the Mechanics and Physics of Solids 40 739-765.

[24] T.C.T. Ting (1996) "Anisotropic Elasticity-Theory and Applications". Oxford University Press, New York. 